() 2019 НМИЦ дгоИ

Поступила 15.03.2019 Принята к печати 10.06.2019

Контактная информация: Федорова Дарья Викторовна, врачгематолог консультативного отделения НМИЦ детской гематологии, онкологии и иммунологии им. Дмитрия Рогачева Минздрава России.

Адрес: 117997, Москва, ГСП-7, ул. Саморы Машела, 1 E-mail: daria.fedorova@fccho-moscow.ru

(c) 2019 by NMRC PHOI

Received 15.03.2019 Accepted 10.06.2019

Correspondence: Daria V. Fedorova, MD, hematologist of Outpatient Consultative Unit, Dmitriy Rogachev National Medical Research Center of Pediatric Hematology, Oncology, Immunology, Ministry of Healthcare of Russian Federation.

Address: Russia 117997, Moscow, Samory Mashela st., 1 E-mail: daria.fedorova@fccho-moscow.ru
ANKRD26-связанная тромбоцитопения как частный случай наследственной тромбоцитопении с предрасположенностью к гематологическим злокачественным новообразованиям

\author{
Я.А. Ердомаева, Д.В. Федорова, П.А. Жарков, М.А. Курникова, С.Г. Манн, Е.В. Райкина
}

ФГБУ «Национальный медицинский исследовательский центр детской гематологии, онкологии и иммунологии им. Дмитрия Рогачева» Минздрава России, Москва

Семейная ANKRD26-связанная тромбоцитопения (ранее известная как тромбоцитопения-2) редкая форма наследственной тромбоцитопении, характеризующаяся низким риском геморрагических осложнений и предрасположенностью к развитию злокачественных заболеваний крови. Пациентам с ANKRD26-связанной тромбоцитопенией и их родственникам требуются генетические консультации, последующее наблюдение и выбор оптимального лечения в случае развития острого лейкоза и других злокачественных заболеваний крови. В статье представлены описание клинического наблюдения данной патологии у двух сиблингов и анализ доступной литературы, посвященной патогенетическим аспектам и тактике ведения пациентов с этим редким заболеванием. Кроме того, рассмотрены общие вопросы диагностики и терапии у пациентов с заболеваниями из группы наследственных тромбоцитопений с предрасположенностью к развитию злокачественных заболеваний крови. Родители пациентов дали согласие на использование инорормации о ребенке в научных исследованиях и публикациях.

Ключевые слова: дети, геморрагический синдром, семейная тромбоцитопения, наследственная патология тромбоцитов, синдромы наследственной предрасположенности к злокачественным заболеваниям, ANKRD26

Ердомаева Я.А. и соавт. Вопросы гематологии/онкологии и иммунопатологии в педиатрии, 2019; 18 (3): 54-61. DOI: 10.24287/1726-1708-2019-18-3-54-61

\section{ANKRD26-related thrombocytopenia: case report and literature review of inherited thrombocytopenias with predisposition to malignancies}

\author{
Ya.A. Erdomaeva, D.V. Fedorova, P.A. Zharkov, M.A. Kurnikova, S.G. Mann, E.V. Raykina \\ Dmitriy Rogachev National Medical Research Center of Pediatric Hematology, Oncology, \\ Immunology Ministry of Healthcare of Russian Federation. Moscow
}

ANKRD26-related thrombocytopenia (previously known as thrombocytopenia-2) is a rare form of inherited platelet disorders. Patients with ANKRD26-related thrombocytopenia usually do not suffer from severe bleeding but have predisposition to acute myeloid leukemia and other malignancies. Patients with ANKRD26-related thrombocytopenia and their relatives need genetic consultation and long term follow-up in view of risk of malignant blood disorders. The clinical case of ANKRD26-related thrombocytopenia in two siblings is presented in this paper. Review of literary data on pathogenesis, treatment and follow-up of patients with ANKRD26-related thrombocytopenia is performed. Common questions of diagnosis and management in patients with congenital thrombocytopenias with predisposition to malignant blood disorders are also reviewed.

Parents gave their permission for using personal data for clinical research and publications.

Key words: children, bleeding, congenital thrombocytopenia, inherited platelet disorders, germline predisposition to malignant disorders, ANKRD26

Erdomaeva Ya.A., et al. Pediatric hematology/oncology and immunopathology, 2019; 18 (3): 54-61. DOI: 10.24287/1726-1708-2019-18-3-54-61
3 а последние 15 лет знания в области наследственных тромбоцитопений (НТ) значительно расширились. На сегодняшний день в группу НТ входит более 30 заболеваний, вызванных мутациями более чем в 25 генах и характеризующихся различной степенью тяжести тромбоцитопении и большой вариабельностью прогноза [1]. Постановка точного диагноза НТ затруднительна по многим причинам. Во-первых, как и в случае многих других редких болезней, врачи склонны подозревать наиболее 
распространенные заболевания, характеризующиеся тромбоцитопенией. Во-вторых, для постановки точного диагноза требуются сложные лабораторные методы диагностики, которые доступны только в крупных специализированных центрах. Как следствие, многим пациентам с НТ устанавливают неправильный диагноз и назначают неэффективное лечение [2]. При этом сохраняется проблема недостаточной диагностики НТ, связанной с низкой эффективностью сбора семейного анамнеза. После комплексного диагностического обследования примерно у 40-50\% пациентов с выраженной картиной НТ диагностика по-прежнему вызывает трудности, поскольку у этих пациентов имеются генетические заболевания, но точный молекулярный дефект еще не выявлен [3].

Тщательный анализ данных сотен пациентов с НТ показал, что, имея различные формы НТ, они подвержены риску развития различных заболеваний в детском или взрослом возрасте, в том числе жизнеугрожающих. В частности, при некоторых формах НТ выше риск развития злокачественных заболеваний крови, при других формах повышен риск развития почечной недостаточности, катаракты и потери слуха, есть также НТ с предрасположенностью к развитию синдрома недостаточности костного мозга $[1,4]$. В связи с этим данные пациенты и их родственники требуют соответствующего генетического консультирования и тщательного наблюдения. Своевременная идентификация пациентов с НТ и определение конкретной нозологической формы важны для оценки прогноза пациентов и их родственников, проведения генетических консультаций, персонализации последующего наблюдения и выбора оптимального лечения в случае развития дополнительных заболеваний.

По нашим данным, на сегодняшний день в отечественной литературе нет описаний ANKRD26-связанной тромбоцитопении. Ниже мы описываем семью с ANKRD26-связанной тромбоцитопенией. Родители пациентов и взрослые больные дали согласие на использование их данных для иследования и публикации.

\section{Клиническое наблюдение}

Пациентка И., 2011 г.р., развивалась согласно возрасту, пороков внутренних органов и внешнего дисморфизма не было. В общем анализе крови (ОАК) в первые сутки жизни количество тромбоцитов $230 \times 10 \%$ л; позже этот показатель в гемограмме регулярно не исследовали. С 4-летнего возраста у девочки отмечен геморрагический синдром в виде легкого появления множественных крупных экхимозов на ногах. В возрасте 4,5 года впервые выявлено снижение тромбоцитов до $58 \times 10 \%$ л; изменения количества или морфологии других клеточных элементов крови не отмечали. По данным миелограм- мы, выполненной по месту жительства, выявлено небольшое количество мегакариоцитов со слабой «отшнуровкой» тромбоцитов. Учитывая кожный геморрагический синдром и тромбоцитопению, ребенку был выставлен диагноз «иммунная тромбоцитопения» (ИТП). Специфическую терапию не назначали в связи с умеренной выраженностью тромбоцитопении и отсутствием клинически значимых кровотечений. В дальнейшем сохранялись тромбоцитопения в пределах 44-79 × 10\%/л, умеренный кожный геморрагический синдром в виде посттравматических экхимозов и петехий, редко - носовые кровотечения.

В возрасте ребенка 5 лет родители обратились за консультацией гематолога в консультативное отделение НМИЦ детской гематологии, онкологии и иммунологии им. Дмитрия Рогачева Минздрава России (далее - НМИЦ ДГОИ) с жалобами на легкое появление экхимозов, периодические носовые кровотечения. При осмотре были выявлены единичные петехиальные элементы на коже туловища и конечностей, единичные экхимозы на коже нижних конечностей. Семейный анамнез показал, что отец ребенка наблюдается у гематолога с гормонрезистентной формой хронической ИТП. Диагноз был установлен в возрасте 18 лет; количество тромбоцитов сохраняется на уровне $30 \times 10 \%$ л (в более раннем возрасте этот показатель достоверно не известен); периодически беспокоят носовые кровотечения (обильные носовые кровотечения отмечались и в детском возрасте); также имели место длительные (до нескольких суток) кровотечения после удаления зубов. У бабушки по линии отца в зрелом возрасте обнаружили тромбоцитопению - $58 \times 10 \%$ л; длительность ее неизвестна. В семье есть два младших сына, 2014 г.р., разнояйцевые близнецы. У одного брата (пациент М.) тромбоцитопению диагностировали в возрасте 1,5 года (количество тромбоцитов - около $30 \times 10 \%$ л); второй брат здоров.

По результатам обследования в НМИЦ ДГОИ у пациентки И. в ОАК подтверждена тромбоцитопения - до $46 \times 10 \%$ л; имеются легкая гипохромия и микроцитоз эритроцитов на фоне нормальной концентрации гемоглобина. По результатам морфологии тромбоцитов повышено количество микроформ. При исследовании функциональной активности тромбоцитов (ФАТ) методом цитофлуориметрии с активацией изменений не выявлено. По результатам миелограммы из двух точек оба пунктата - со сниженной клеточностью, полиморфные, сходные между собой по составу, нейтральный жир в умеренном количестве. Мегакариоцитарный росток расширен, отмечается скудная «отшнуровка» тромбоцитов в 3-5\% мегакариоцитов. Остальные ростки кроветворения сохранены. Цитогенетических нарушений в клетках костного мозга не выявлено. Учитывая отягощенный 
семейный анамнез, пациентке И. методом высокопроизводительного секвенирования ДНК из клеток перефирической крови был произведен поиск патогенных мутаций, ассоциированных с тромбоцитопениями, тромбоцитопатиями, коагулопатиями (таргетная панель «Тромбоцитопении, тромбоцитопатии»). В гене ANKRD26 в 5'-нетранслируемой области была обнаружена замена одного нуклеотида в гетерозиготном состоянии с.-118C > A, описанная в литературе как патогенная при ANKRD26-связанной тромбоцитопении [4]. Данная замена подтверждена методом прямого секвенирования по Сэнгеру.

На основании полученных данных пациентке поставлен диагноз: семейная (наследственная) ANKRD26-связанная тромбоцитопения (ANKRD26 c.$118 \mathrm{C}>\mathrm{A}$ ).

Через год от начала обследования пациентки И. было проведено комплексное обследование пациента М. в условиях НМИЦ ДГОИ. Из анамнеза известно, что впервые тромбоцитопения (около $30 \times 10^{9} /$ л) обнаружена у него в возрасте 1,5 года; в это же время родители отмечали у ребенка склонность к появлению экхимозов, периодические необильные носовые кровотечения, однократно - субконъюктивальное кровоизлияние. При осмотре был выявлен умеренный кожный геморрагический синдром в виде многочисленных экхимозов диаметром до 1 см на конечностях, единичные - на коже туловища. По результатам дополнительных обследований: тромбоцитопения - до $52 \times 10 \%$ л; выраженных изме-

\section{Рисунок 1}

Генеалогическое древо семьи

(стрелкой указан пробанд - пациентка И.)

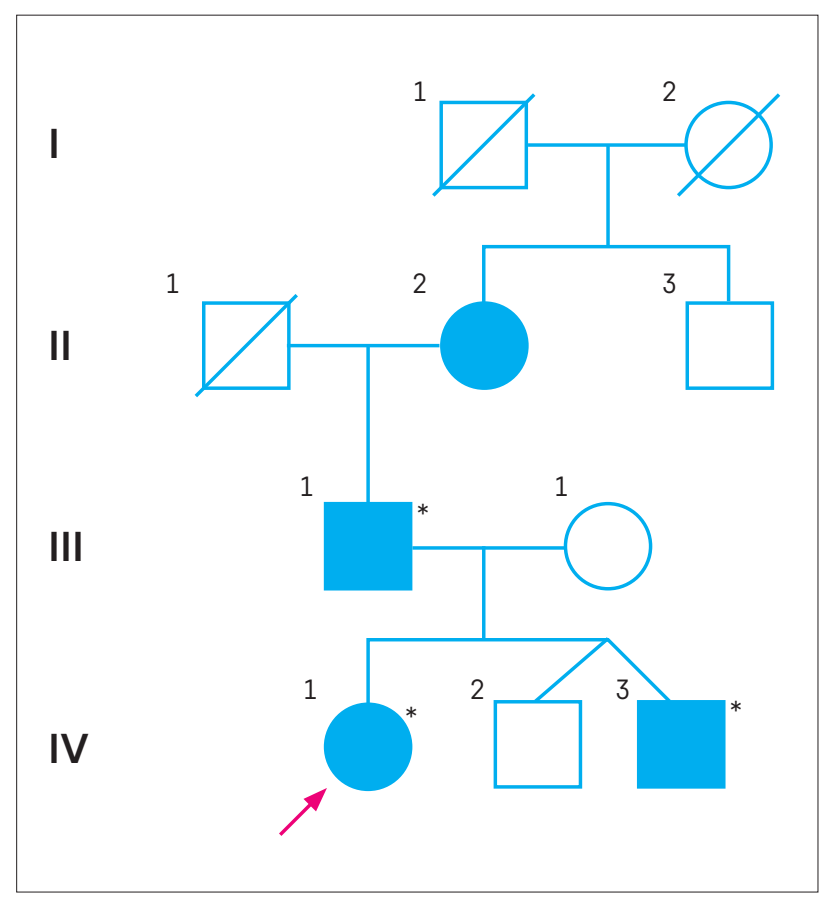

* Члены семьи, которым было выполнено молекулярно-генетическое исследование. нений морфологии или функциональной активности тромбоцитов не выявлено.

По результатам генетического исследования отца детей и пациента М. была выявлена патогенная мутация в гене $A N K R D 26$ методом секвенирования по Сэнгеру. Верифицирован диагноз: семейная (наследственная) ANKRD26-связанная тромбоцитопения (замена с.-118C > А в гене ANKRD26). Случаи ОМЛ, МДС или других гематологических злокачественных заболеваний в семье не наблюдались (рисунок 1). Семье были даны общие рекомендации по профилактике и лечению кровотечений, подготовке к инвазивным процедурам и стоматологическому лечению, рекомендовано динамическое наблюдение у педиатра и гематолога.

\section{ОБЗОР ЛИТЕРАТУРЫ}

Диагностика НТ. Диагностический поиск конкретной формы НТ можно схематически разделить на два больших этапа. Первый этап - отбор пациентов, вероятнее всего, имеющих генетическую форму, среди общего числа пациентов с изолированной тромбоцитопенией. Второй этап - поиск конкретного генетического расстройства.

При дифференциальной диагностике пациентов с количеством тромбоцитов ниже $150 \times 10 \%$ л необходимо учитывать возможность наследственного генеза данной тромбоцитопении, особенно в случаях, когда невозможно установить количество тромбоцитов в более ранних анализах крови с момента рождения. Доказано, что существует вариабельность нормального диапазона количества тромбоцитов, связанная с гендерными, возрастными, а также этническими характеристиками. У здоровых людей данная вариабельность в значительной степени ответственна за физиологическое снижение количества тромбоцитов (< $150 \times 10^{9} /$ л) [5]. Требуется тщательная оценка клинической картины и семейного анамнеза у пациентов с тромбоцитопенией легкой степени тяжести.

Часто при НТ умеренная тромбоцитопения обнаруживается случайно у бессимптомного пациента при проведении планового ОАК в случае отсутствия в течение жизни ситуаций повышенного риска развития значимой кровоточивости, таких как операция, удаление зуба, роды или прием препаратов, вызывающих дисфункцию тромбоцитов (например, аспирина). В детской гематологии чаще встречаются пациенты с синдромальными формами НТ, ассоциированными с врожденными дефектами.

Отсутствие данных о том, что у других членов семьи имеется тромбоцитопения, не исключает наследственную причину, поскольку патогенетические мутации могут возникать de novo, а некоторые на- 
следственные тромбоцитопении наследуются рецессивно. Также целесообразно уточнить наличие у членов семьи заболеваний, риск развития которых может быть повышен при отдельных формах НТ. В случае форм с аутосомно-доминантным типом наследования важно помнить о явлении неполной пенетрантности мутантного гена. В литературе описаны случаи нормального количества тромбоцитов у носителей мутации в семьях с различными формами НТ [3].

Исследование мазка периферической крови обязательно при первоначальной оценке всех пациентов с тромбоцитопенией и может выявить характерные аномалии тромбоцитов, эритроцитов или лейкоцитов. Оценка размера тромбоцитов - ключевой инструмент дифференциальной диагностики наследственной и приобретенной тромбоцитопении, а также она важна при проведении дифференциальной диагностики различных форм НТ. Для дифференциальной диагностики с ИТП используют автоматический подсчет фракции незрелых тромбоцитов (\%IPF): повышенние показателя \%IPF связано с усилением деструкции тромбоцитов в периферической крови.

Существуют также специфические лабораторные тесты для диагностики конкретных форм НТ. Тест агрегации тромбоцитов с ристоцетином и проточная цитометрия позволяют поставить диагноз «синдром Бернарда-Сулье, биаллельная форма», а также «болезнь Виллебранда тромбоцитарного типа». Иммунофлуоресцентный анализ белка МҮН9 на предметных стеклах позволяет легко распознать типичные белковые агрегаты в нейтрофилах, которые присутствуют у пациентов с МҮН9-связанной тромбоцитопенией. Данный тест показал 100\%-ю чувствительность и 95\%-ю специфичность для диагностики МҮН9-связанной тромбоцитопении [6].

Современные методы высокопроизводительного секвенирования ДНК для параллельного скрининга различных мутаций в ближайшем будущем могут стать основным методом диагностики НТ, поскольку становятся все более эффективными и менее дорогостоящими. Таким образом, одношаговое секвенирование всех потенциальных генов в качестве базового диагностического подхода у пациентов с подозрением на НТ может оказаться более экономически эффективным, чем применение широкого спектра различных лабораторных тестов с дальнейшим исследованием наиболее вероятных генов для анализа.

\footnotetext{
HT с предрасположенностью к гематологическим злокачественным новообразованиям. Впервые наследственный характер развития острых лейкозов был описан в 1922 году, но первое молекулярное объяснение наследственной предрасположенности к острому миелоидному лейкозу (ОМЛ)
}

было получено после открытия в 1999 году патогенной герминальной мутации гена RUNX1 [7, 8].

На сегодняшний день выделяют три наследственные формы тромбоцитопении с доказанной предрасположенностью к гематологическим злокачественным новообразованиям: семейная тромбоцитопения с предрасположенностью к ОМЛ (FPD/AML - от англ. familial platelet disorder with propensity to acute myeloid leukemia), ANKRD26-связанная тромбоцитопения и ETV6-связанная тромбоцитопения [1]. В отечественной литературе описаны две семьи из России c FPD/AML $[9,10]$. Последний пересмотр классификации миелоидных новообразований и острых лейкозов В03 от 2016 года подтвердил существование этой группы НТ и ввел новую категорию заболеваний, определяемых как «миелоидные новообразования с наследственной предрасположенностью и предсуществующими тромбоцитопениями» [11]. К сожалению, это определение не точное, потому что у пациентов c ETV6-связанной тромбоцитопенией в большинстве случаев развивается острый лимфобластный лейкоз (ОЛЛ).

Данная группа НТ обладает рядом важных общих характеристик, таких как аутосомно-доминантный тип наследования, тромбоцитопения легкой и средней степени тяжести и нормальный (или почти нормальный) размер тромбоцитов. Более того, при всех трех заболеваниях по результатам исследования костного мозга определяется нормальное или увеличенное количество мегакариоцитов, как правило, с признаками дисплазии в виде гиполобулированных ядер небольших размеров и скудной «отшнуровкой» тромбоцитов. Другие ростки кроветворения исходно не поражены. В связи с наличием дисмегакариопоэза описаны случаи ошибочной постановки диагноза миелодиспластического синдрома (МДС), форма рефрактерной цитопении с однолинейной дисплазией (рефрактерная тромбоцитопения), согласно классификации миелоидных новообразований В03 2008 года. Однако диагноз «МДС с рефрактерной тромбоцитопенией» встречается крайне редко (до 0,1-2\% случаев) [2]. Таким образом, в случае обнаружения дисмегакариопоэза у пациента с изолированной тром- боцитопенией диагноз НТ следует рассматривать как гораздо более вероятный, чем диагноз МДС.

С другой стороны, клинически значимой разницей при дифференциальной диагностике является сохранение нормальной функции тромбоцитов при ANKRD26-связанной тромбоцитопении и ETV6-связанной тромбоцитопении, в отличие от сложного дефекта тромбоцитов при FPD/AML, которая приводит к нарушению агрегации тромбоцитов и высвобождения плотных гранул, а иногда и к тяжелой кровоточивости, не соответствующей выраженности тромбоцито- 
пении. Вероятнее всего, это связано с тем, что мутация в гене RUNX1 при FPD/AML вызывает нарушение регуляции экспрессии легкой цепи миозина тромбоцитов, приводящей к тромбоцитопатии [12].

Тем не менее отметим, что все перечисленные генетические дефекты ведут к нарушению баланса между пролиферацией и дифференцировкой кроветворных предшественников за счет усиленной пролиферации. К тому же при острых лейкозах часто обнаруживают соматические мутации в гене RUNX1, а также в гене ETV6, реже - в гене ANKRD26, что подтверждает роль RUNX1, ANKRD26 и ETV6 в развитии и семейных, и спорадических острых лейкозов и МДС.

ANKRD26-связанную тромбоцитопению (тромбоцитопению-2) вызывают моноаллельные точечные мутации в 5'-нетранслируемой области гена ANKRD26, функция которого до конца не известна. Мутации в 5'-нетранслируемой области гена ANKRD26 ответственны за дефектное связывание факторов транскрипции RUNX1/FLI1 с геном ANKRD26, что приводит к отсутствию снижения экспрессии этого гена в зрелых мегакариоцитах, которое наблюдается в норме. Постоянная экспрессия ANKRD26 в зрелых мегакариоцитах сопровождается усилением сигнального пути TPO/MPL и нижестоящего сигнального пути MARK/ERK [13]. Важно отметить, что данная гипотеза была подтверждена коррекцией дефекта «отшнуровки» тромбоцитов В клеточной линии мегакариоцитов пациентов с
ANKRD26-связанной тромбоцитопенией на фоне использования ингибитора МЕК [13]. Следовательно, наблюдаемую гиперактивацию пути MARK/ERK у пациентов с ANKRD26-связанной тромбоцитопенией можно будет рассматривать в будущем как терапевтическую мишень для контроля тромбоцитопении. Интересно отметить, что подобный дефект мегакариопоэза на поздних стадиях наблюдается у пациентов с FPD/AML [7]. Сигнальный путь TPO/MPL регулирует не только мегакариопоэз, но также поддержание пула гемопоэтических стволовых клеток (ГСК) на протяжении жизни. Вероятно, ANKRD26опосредованная дизрегуляция сигнальных путей в ГСК способствует лейкемической трансформации [14]. Доказано, что активация сигнального пути MARK приводит к увеличению пролиферации и выживаемости злокачественных клеток при раке молочной железы, раке толстой кишки, меланоме и немелкоклеточном раке легких [15].

По-видимому, ANKRD26-связанная тромбоцитопения - одна из наиболее частых форм НТ. В итальянском исследовании 274 семей с НТ ANKRD26-связанная тромбоцитопения была обнаружена у 20\% пациентов с подтвержденным молекулярным диагнозом [16]. При дальнейшем исследовании выявленных пациентов с ANKRD26-связанной тромбоцитопенией (222 случая) было обнаружено, что у них существует повышенный риск развития миелоидных злокачественных новообразований: у 4,9\%

\section{Рисунок 2}

Патогенез развития тромбоцитопении у пациентов с мутациями в гене ANKRD26 [13]

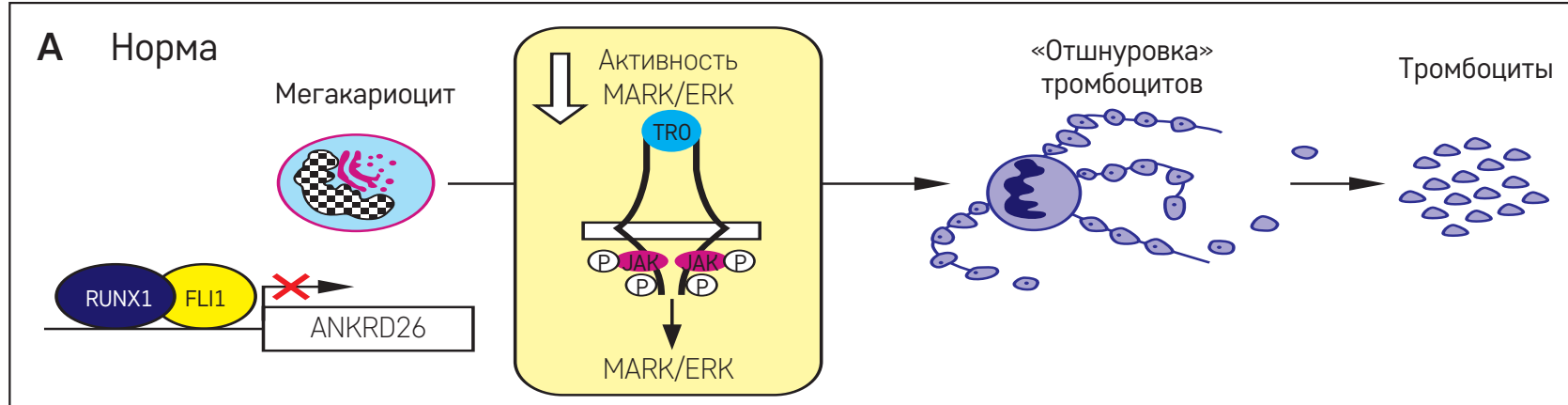

Б $\quad$ ANKRD26-HTח

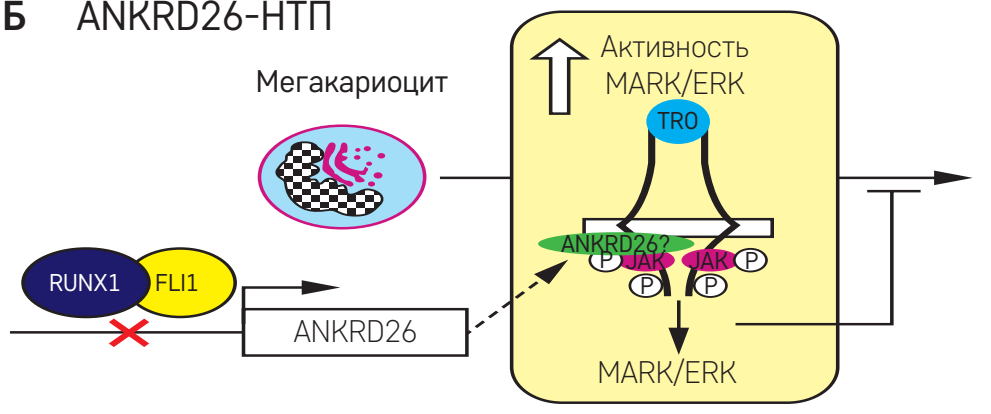

Десрект «отшнуровки» тромбоцитов

Тромбоциты
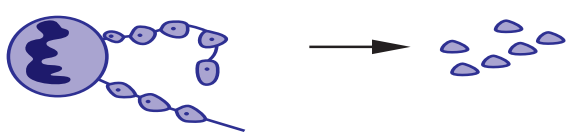

Примечания: $\boldsymbol{A}$ - у здорового человека комплекс RUNX1/FLI1 негативно регулирует экспрессию гена ANKRD26 в мегакариоцитах, прогрессивно снижая активность пути TPO/MPL/MAPK/ERK во время дифференцировки мегакариоцитов, в результате этого происходит «отшнуровка» тромбоцитов; Б - у пациентов с ANKRD26-связанной тромбоцитопенией мутации в 5'-UTR гена ANKRD26 нарушают процесс ингибирования RUNX1/FLII, что приводит к патологической экспрессии ANKRD26 в мегакариоцитах. ANKRD26 накапливается во внутренней части клеточной мембраны и опосредованно, через TPO/MPL, приводит к гиперактивации пути MAPK/ERK с последующим глубоким дефектом в образовании тромбоцитов. 
пациентов развился ОМЛ (возраст манифестации 40-60 лет); у 2,2\% - МДС (возраст манифестации 35-70 лет) и у $1,3 \%$ - хронический миелолейкоз (ХМЛ) (возраст манифестации - 30-65 лет). По результатам ретроспективного исследования данной выборки пациентов, частота встречаемости ОМЛ, МДС и ХМЛ составила 123 (ДИ: 62-221), 56 (ДИ: 18131) и 34 (ДИ: 7-98) соответственно на 100 тыс. населения и оказалась значительно выше, чем частота встречаемости ОМЛ, МДС и ХМЛ в общей популяции (5,2; 4,5 и 1,6 на 100 тыс. населения, по данным Национального института онкологии США). Таким образом, у 8\% пациентов с ANKRD26-связанной тромбоцитопенией было отмечено развитие злокачественного заболевания крови [17] (рисунок 2).

\section{Алгоритм ведения пациентов с НТ с предраспо-} ложенностью к гематологическим злокачественным новообразованиям. Распространенность наследственных гемобластозов на сегодняшний день точно не известна; предполагается, что по меньшей мере у 4-13\% пациентов до 18 лет и 5\% взрослых пациентов ОМЛ/МДС носит наследственный характер [18].

Оптимальный подход к клиническому наблюдению пациентов с герминальными мутациями, ассоциированными с развитием острого лейкоза и других миелопролиферативных заболеваний, неизвестен. Согласно рекомендациям отдела по оценке риска развития острого лейкоза Чикагского университета, первоначальная базовая гематологическая оценка должна включать OAK, HLA-типирование и пункцию костного мозга (КМ) с цитогенетическим исследованием клеток КМ [19]. При отсутствии лабораторных изменений пациенты сдают ОАК каждые 6-12 мес.; в случае выявления отклонений в ОАК проводится повторная пункция КМ с цитогенетическим исследованием КМ. Если в костном мозге нет признаков поражения, то повторный ОАК следует выполнять ежемесячно, а повторную пункцию КМ - через 3-4 мес. по аналогии с рекомендациями по наблюдению пациентов с врожденными аплазиями КМ. Другие авторы в качестве первоначальной гематологической оценки предлагают только проведение ОАК [18].

Особенности лечения пациентов с НТ с развитием клонального поражения костного мозга.

Большинство экспертов считает, что пациентам с острым лейкозом, возникшим на фоне наследственной предрасположенности, целесообразно проводить аллогенную трансплантацию гемопоэтических стволовых клеток (ТГСК) независимо от прогностических маркеров с использованием клеток донора, у которого доказано отсутствие герминальной мутации. Данный подход обоснован необходимостью полной эрадикации не только злокачественного клона кро- ветворения, но и нормального клона кроветворения с предрасположенностью к гемобластозам [18, 19]. Данный подход не является универсальным и требует дополнительных исследований. При этом следует отметить, что ОМЛ у пациентов с герминальной мутацией гена СЕВРА характеризуется высокой химиочувствительностью и риском развития последующих первичных миелоидных злокачественных новообразований, которые обычно называют «рецидивами», но они таковыми не являются [18]. Другие наследственные формы острых лейкозов могут обладать аналогичными характеристиками с точки зрения химиочувствительности и риска последующих первичных острых лейкозов или МДС [18, 19].

Лечащим врачам необходимо заблаговременно приступать к поиску подходящего донора для аллогенной ТГСК для пациентов с НТ с предрасположенностью к гематологическим злокачественным новообразованиям, которым планируется проведение аллогенной ТГСК. Всякий раз, когда доступен родственный донор, крайне важно исключить наличие у него герминальной мутации, поскольку ТГСК от родственного донора может привести к повторному развитию острого лейкоза из донорских клеток. Теоретически риск развития острого лейкоза/МДС у родственного донора потенциально увеличивается после процедуры мобилизации стволовых клеток. у таких доноров зачастую отмечают также высокий риск неудачного афереза стволовых клеток; примерно в 7\% случаев неудачи связаны с наличием герминальной мутации линии $[18,20]$.

Особенности верификации диагноза НТ у пациентов с клональным поражением костного мозга. Зачастую диагноз НТ с предрасположенностью к гематологическим злокачественным новообразованиям устанавливают после диагностики острого лейкоза или МДС. В первую очередь это связано с легкой степенью тромбоцитопении и отсутствием кровоточивости у пациента. В последнее время после пересмотра классификации миелоидных новообразований В03 от 2016 года диагностика наследственных синдромов с предрасположенностью к ОМЛ/МДС была включена в большинство стандартных диагностических алгоритмов. Для центров, проводящих генетическое тестирование опухолевых клеток на мультигенной панели высокопроизводительного секвенирования, включающей гены RUNX1, ANKRD26, ETV6, эксперты советуют проводить оптимизацию таких тестов для обнаружения всех вариантов герминальных мутаций в этих генах и стремиться к инициальному генетическому исследованию опухолевых клеток и здоровых клеток для максимального выявления и оптимизации лечения пациентов с герминальными мутациями [21]. 
Основной материал для генетического тестирования на наличие герминальных мутаций - периферическая кровь (ПК), однако при гемобластозах ПК содержит опухолевые клетки. Возрастные клональные изменения гемопоэза также усложняют анализ ПК, так как у 1\% здоровых пациентов в возрасте до 50 лет и у 10\% здоровых пациентов в возрасте 65 лет могут иметься мутации в генах, связанные с наследственными синдромами, с предрасположенностью к ОМЛ/МДС. Таким образом, врачи должны избегать использования ПК, КМ или других тканей, контактирующих с ПК, таких как слюна, буккальные клетки. По мнению ряда экспертных групп, предпочтительным источником ДНК для обнаружения герминальной мутации у пациентов с острым лейкозом или МДС является ДНК из культивируемых фрибробластов кожи, полученных с помощью 3-миллиметровой биопсии кожи или путем удаления кожного эллипса при проведении трепанобиопсии КМ. Основной недостаток использования культивируемых фиибробластов кожи - период времени до 3-6 нед., необходимый для культивирования достаточного количества клеток для генетического тестирования, а также отсутствие технических возможностей для культивирования фиибробластов кожи в ряде центров.

\section{ЗАКЛЮЧЕНИЕ}

В последние годы группа НТ превратилась в крайне гетерогенную категорию заболеваний, включающую синдромальные формы с поражением различных органов и тканей. С клинической точки зрения это подразумевает необходимость междисциплинарного подхода к ведению пациентов с НТ, принимая во внимание не только их склонность к кровотечениям, но и врожденные синдромальные расстройства и риск развития других заболеваний в будущем. Лучшее понимание механизма возникновения тромбоцитопении и других заболеваний дает уникальную возможность улучшить наши знания не только в области мегакариопоэза, но и в других медицинских областях, таких как злокачественная клональная пролиферация клеток крови, недостаточность костного мозга, поражение почек, слуха и зрения.

Важно отдельно выделить группу НТ с предрасположенностью к гематологическим злокачественным новообразованиям в связи с особенностями ведения таких пациентов в случае развития острого лейкоза или МДС. Врач-гематолог должен быть осведомлен о группе наследственных синдромов с предрасположенностью к гематологическим злокачественным новообразованиям и направлять всех пациентов с подозрительным семейным анамнезом, характерными синдромальными проявлениями для генетического консультирования и генетического исследования на наличие герминальной мутациии. Данные пациенты должны наблюдаться в центре, специализирующемся на ведении больных с наследственными формами острых лейкозов/МДС. Крайне важно, чтобы родственные совместимые доноры ГСК были тщательно оценены на наличие герминальной мутации, чтобы избежать потенциальной возможности вторичного развития гемобластоза у реципиента или повышения риска развития лейкоза у родственного донора из-за мобилизации стволовых клеток.

На сегодняшний день остается много вопросов, требующих дальнейших исследований. Крупные всесторонние исследования носителей герминальной мутации вне зависимости от инициального статуса КМ, включающие молекулярную характеристику до и во время клональной эволюции, позволят определить риск развития острого лейкоза, влияние соматических мутаций и эпигенетических аберраций, а также факторов окружающей среды и других фракторов, которые могут способствовать клональной эволюции и развитию острого лейкоза. Исследования в данной области позволят в конечном итоге разработать эфффективные методы профилактики и алгоритмы ведения пациентов, аналогичные прогрессивным методикам лечения пациенток с наследственным раком молочной железы и яичников.

Активно обсуждаются следующие вопросы:

- идентификация новых генов, герминальные мутации в которых приводят к развитию НТ или к предрасположенности к гематологическим злокачественным новообразованиям;

- распространенность НТ и наследственных гематологических злокачественных новообразований в детской и взрослой популяциях;

- целесообразность инициального исследования на наличие герминальных мутаций у пациентов с острым лейкозом/МДС;

- оптимальный источник ДНК для определения герминальной мутации;

- роль профилактической аллогенной ТГСК, в частности, при развитии цитопении неясного значения.

\section{ИСТОЧНИК ФИНАНСИРОВАНИЯ}

Не указан.

\section{КОНФЛИКТ ИНТЕРЕСОВ}

Авторы статьи подтвердили отсутствие конфликта интересов, о котором необходимо сообщить

ORCID

Erdomaeva Ya.A. ORCID: http://orcid.org/0000-0002-3810-1398

Fedorova D.V. ORCID: http://orcid.org/0000-0003-4567-1871

Zharkov P.A. ORCID: http://orcid.org/0000-0003-4384-6754

Kurnikova M.A. ORCID: http://orcid.org/0000-0003-0900-6874

Mann S.G. ORCID: https://orcid.org/0000-0002-1014-5196

Raykina E.V. ORCID: http://orcid.org/0000-0002-7634-2053 


\section{Литература}

1. Melazzini F., Zaninetti C., Balduini C.L. Bleeding is not the main clinical issue in many patients with inherited thrombocytopaenias. Haemophilia 2017; 23: 673-81.

2. Zaninetti C., Santini V., Tiniakou M., Barozzi S., Savoia A., Pecci A. Inherited thrombocytopenia caused by ANKRD26 mutations misdiagnosed and treated as myelodysplastic syndrome: report on two cases. J Thromb Haemost 2017; 15: 2388-92.

3. Pecci A. Diagnosis and treatment of inherited thrombocytopenias. Clin Genet 2016; 89: 141-53.

4. Noris P., Favier R., Alessi M., Geddis A.E., Kunishima S., Heller P.G., et al. ANKRD26-related thrombocytopenia and myeloid malignancies. Blood 2013; 122 (11): 1987-9.

5. Balduini C.L., Pecci A., Noris P. Diagnosis and management of inherited thrombocytopenias. Semin Thromb Hemost 2013; 39 (2): 161-71.

6. Kitamura K., Yoshida K., Shiraishi Y., et al. Normal neutrophil myosin IIA localization in an immunofluorescence analysis can rule out MYH9 disorders. J Thromb Haemost 2013; 11: 2071-3.

7. Gunz F.W., Gunz J.P., Veale A.M., Chapman C.J., Houston I.B. Familial leukaemia: a study of 909 families. Scand J Haematol 1975; 15 (2): 117-31.

8. Song W.J., Sullivan M.G., Legare R.D., Hutchings S., Tan X., Kufrin D., et al. Haploinsufficiency of CBFA2 causes familial thrombocytopenia with propensity to develop acute myelogenous leukaemia. Nat Genet 1999; 23 (2): 166-75.
9. Бобрынина В.О., Баранова О.Ю., Самочатова Е.В., Масчан А.А. Семейная тромбоцитопения/тромбоцитопатия с предрасположенностью к развитию острого миелоидного лейкоза: описание новой семьи и мутации в гене RUNX1. Онкогематология 2011; 4: 6-11.

10. Хаджиева Ф.Р., Жарков П.А., Федорова Д.В., Райкина Е.В., Игнатова А.А., Плясунова С.А. и др. Случай редкой наследственной тромбоцитопении с предрасположенностью к развитию острого миелоидного лейкоза у детей-близнецов. Вопросы гематологии/онкологии и иммунопатологии в педиатрии 2018; 17 (4): 51-6.

11. Arber D.A., Orazi A., Hasserjian R., Thiele J., Borowitz M.J., Le Beau M.M., et al. The 2016 revision to the World Health Organization classification of myeloid neoplasms and acute leukemia. Blood 2016; 127 (20): 2391-405.

12. Balduini C.L., Melazzini F., Pecci A. Inherited thrombocytopenias - recent advances in clinical and molecular aspects. Platelets 2016; 28 (1): 3-13.

13. Bluteau D., Balduini A., Balayn N., Currao M., Nurden P., Deswarte C., et al. Thrombocytopenia-associated mutations in the ANKRD26 regulatory region induce MAPK hyperactivation. J Clin Invest 2014; 124: 580-91.

14. de Graaf C.A., Metcalf D. Thrombopoietin and hematopoietic stem cells. Cell Cycle 2011; 10 (10): 1582-9.

15. Wang D., Boerner S.A., Winkler J.D., Lorusso P.M. Clinical experience of MEK inhibitors in cancer therapy. Biochim Biophys Acta 2007; 1773 (8): 1248-55.
16. Noris P., Perrotta S., Seri M., Pecci A., Gnan C., Loffredo G., et al. Mutations in ANKRD26 are responsible for a frequent form of inherited thrombocytopenia: analysis of 78 patients from 21 families. Blood 2011; 117: 6673-80.

17. Noris P., Favier R., Alessi M.C., Geddis A.E., Kunishima S., Heller P.G., et al. ANKRD26related thrombocytopenia and myeloid malignancies. Blood 2013; 122: 1987-9.

18. Akpan I.J., Osman A.E.G., Drazer M.W., Godley L.A. Hereditary Myelodysplastic Syndrome and Acute Myeloid Leukemia: Diagnosis, Questions, and Controversies. Curr Hematol Malig Rep 2018; 13: 426-34.

19. Churpek J.E., Godley L.A. How I diagnose and manage individuals at risk for inherited myeloid malignancies. Blood 2016; 128: 1800-13.

20. Rojek K., Nickels E., Neistadt B., Marquez R., Wickrema A., Artz A., et al. Identifying inherited and acquired genetic factors involved in poor stem cell mobilization and donor-derived malignancy. Biol Blood Marrow Transplant 2016; 22 (11): 2100-3.

21. Tawana K., Drazer M.W., Churpek J.E. Universal genetic testing for inherited susceptibility in children and adults with myelodysplastic syndrome and acute myeloid leukemia: are we there yet? Leukemia 2018; 32 (7): 1482-92. 